(REVIEW ARTICLE)

\title{
An analysis of nCovid-19
}

\author{
Khan Sheema Fatima * \\ Shadan Institute of Medical Sciences, Teaching Hospital and Research Center, Hyderabad-86, Telangana, India.
}

Publication history: Received on 02 June 2020; revised on 12 June 2020; accepted on 14 June 2020

Article DOI: https://doi.org/10.30574/wjarr.2020.6.3.0183

\begin{abstract}
The global nCovid-19 pandemic is changing the way we used to live. It is caused by SARS-CoV-2 virus which belongs to a family of coronavirus. These are a group of virus with RNA as their genetic material. The distinctive feature about its structure is the protein spikes which is used to bind to receptors of host cells. It not only causes respiratory distress but also produces severe inflammation and cytokine storm. It forms clot and micro emboli leading to heart attacks and strokes. It also mutates at a rate which is actually slower than the common flu virus. But the pattern of mutation is still not well predicted causing different effects on the infected, thus making diagnosis difficult. So the newly born type of coronavirus, is really a mysterious member of nature, in not only how it originated but also as to how it spread, how it affects the body, recovery and the ways to control it. The aim of this review is describe about the coronavirus as a disease and its symptoms, ongoing treatment methods employed and preventive precautionary steps to be taken by the general public in order to safeguard themselves from this savage contagion.
\end{abstract}

Keywords: SARS-CoV-2; nCovid-19; Pandemic; Pathophysiology; Symptoms and Signs

\section{Briefing about pandemics}

The family of coronavirus has caused various pandemic in World History such as Severe Acute Respiratory Syndrome (SARS) pandemic in 2002, Middle Eastern Respiratory Syndrome (MERS) in 2012.[1,2] so far these diseases were as deadly and contagious as the nCovid-19 but disappeared relatively quickly without causing large number of deaths or producing the urgent the need of a vaccine. The Porcine epidemic diarrhea virus (PDEv) is also a family of coronavirus which caused an epidemic in the year 2013-14 causing an epidemic in pigs leading to huge livestock loses https://www.nationalgeographic.com/news/2014/5/140501-pigs-virus-meat-prices-food-science-health/.Yet, the latter did not cause harm to humans. Some of the species of coronavirus are known to cause common cold [3]. In fact the first strain of coronavirus was obtained from nasal discharge of a patient suffering from common cold in 1970's. [4]

\section{Coronavirus}

\subsection{Structure}

The structure of coronavirus are spherical pleomorphic enveloped viruses with single stranded RNA as their genetic material [3]. They have the largest genome among all RNA virus [5] The characteristic feature of coronavirus is distinctive protein spikes on their exterior surface. These are glycoproteins in somewhat rounded and petal shaped spikes. In electron microscopy these resembles solar corona or crown or halo-like appearance around them [6]. The novel coronavirus belong to family of Coronaviridae in the order Nidovirales [7,8]. There are four genera of coronavirus viz. Alphacoronavirus, Betacoronavirus, Deltacoronavirus, Gammacoronavirus. [5] This nCovid-19 is the seventh member of Coronaviridae and belongs to betacoronavirus genra.[9] Alpha and betacoronavirus are known to infect mammals.[5]

\footnotetext{
* Corresponding author: Khan Sheema Fatima
} 


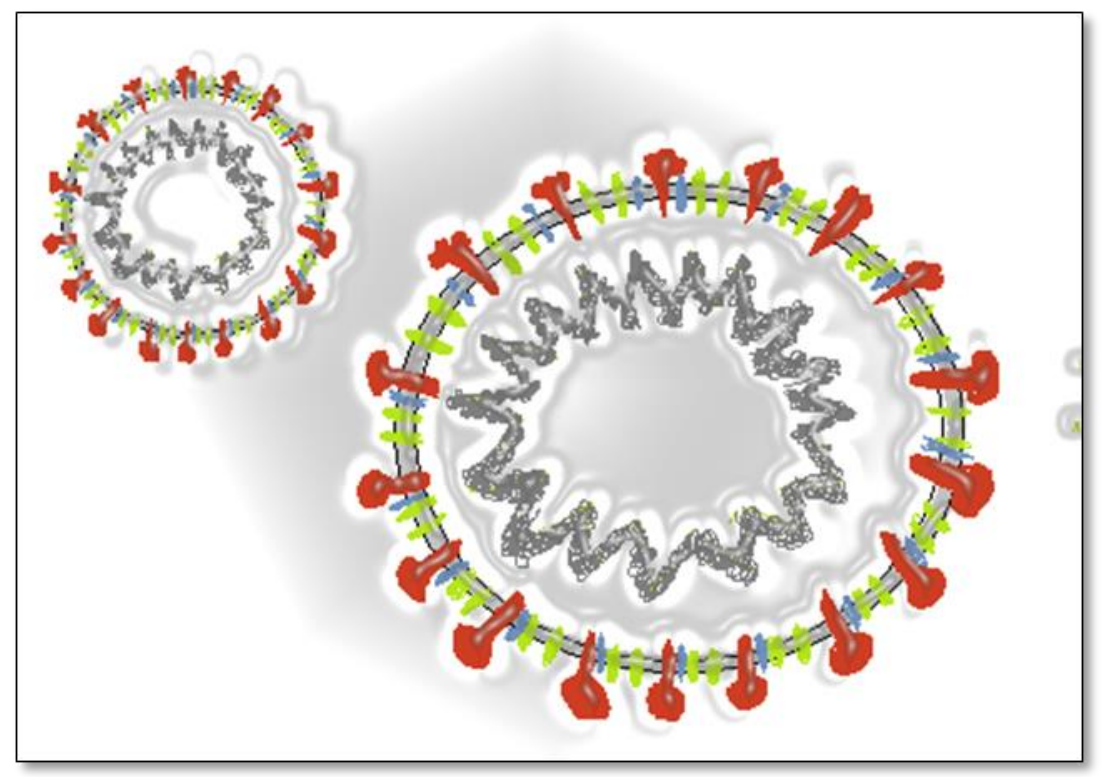

Figure 1 Structure of coronavirus

\subsection{Mutation}

Mutations are defined as changes in base sequence of a genome that causes changes in structure and function of protein synthesized and manifest itself in a way which is considered against the normal. Mutations occurs in all living beings every day. In fact, it what makes us who we are today. It brings about evolution and changes which helps us to adapt to various environmental stress. These are called beneficial mutations. Examples of such mutations are sickle cell anemia. People with sickle cell anemia have shown resistance against malaria disease. Due to sickle like structure of RBC which in turn is caused by formation of hemoglobin fibrils inside it, the malarial parasite does not get a suitable environment to grow and multiply, thereby no infection occurs. While other are waste and degradative, causing loss of life and livelihood. When DNA is the genetic material mutation occurs at far lower rate which is accounted for its stable structure and proofreading capacity. RNA on the other hand is catalytic and due to its unstable structure undergoes increased mutations.

Coronavirus, as previously said, is an RNA virus. But the unique feature of this virus is that is has proofreading capability. It checks for RNA mistakes and can correct them thereby AVOIDING mutations. As a result of which, we see mutation of coronavirus occurs at a rate SLOWER than the common flu virus. Also, the scientist pointed that the mutation occurred so far haven't actually caused an alarming changes. What is interesting to note is that due to slow rate of mutation it might be actually possible to create a vaccine for nCovid-19, whereas for common flu virus and influenza virus we could not create a vaccine for this reason.

\section{How covid-19 pandemic began?}

While the world was busy with their ever important works such as airstrikes in Syria, protest against and for the new bill, or the bombing and shooting taking place in most corner of the world, cutting of forest to create more assets for humans and growing smoggy skies due to fast vehicle mediated life, little did we know that a silent pandemic was growing ferociously strong and fast waiting to unleash the angry storm of nature that we were previously recklessly inflicting on her. The coronavirus pandemic is said to begin in China in early December. In late December 2019 there were cluster of cases reported of undiagnosed pneumonia spreading from Huanan Seafood Wholesale Market in Wuhan [9].

\section{How does coronavirus spread?}

As previously implied out of 100 coronaviruses only 7 infects humans so far. Out of which four causes common cold and they infect usually infect nose or upper respiratory tract and has fast spreading capacity. The other two viruses which are more fatal, namely, SARS-CoV-1 and MERS effects lungs and are spread less. The new SARS-CoV-2 is a combination of both which fast spreading, effects lungs and is highly dangerous. 
Coronavirus spread by touching the contaminated surface and then touching eyes nose and mouth. The protein spikes of virus bind well to cells in these organs. The immune response of the body 'splats' fluid meant for trapping the virus. As a consequence of this fluid, it causes airway obstruction and leads to breathing problem.

\section{Pathophysiology of coronavirus}

The novel SARS-CoV-2 produces a broad spectrum of respiratory disorders. The peculiarity about coronavirus is that, according to the new researches that, the nCovid-19 is not just Acute Respiratory Distress Syndrome, rather is more of an inflammatory disease. While other coronavirus affected pulmonary epithelium, this disease effects pulmonary endothelium. Recent researches suggests coronavirus irritates the endothelium of blood vessels causing the release of fibrin leading to formation of fibrin clot The autopsy done reveals formation of fibrin thrombi. Also, many patients had elevated D-dimer levels (a biomarker seen in high or excess level of clotting) and cutaneous changes in their extremities suggesting thrombotic microangiopathy.[10] Therefore, large number of intravascular emboli and large-vessel thrombosis can be a possible cause of multisystem organ failure as seen in upcoming cases.[11]

\section{Symptoms of coronavirus}

There is a huge list of variable amount of coronavirus symptoms that we are coming across every day. Some of these are similar to common cold but others are highly unique and even rare. Here is a list of early signs and symptoms which are common to all age groups.

\subsection{Flu like symptoms; cough, fever, runny nose, fatigue and headache}

The first symptoms that most people get are runny nose, with prominent cough and fever. These can worse on their own for some while others recovery at fairly fast rate, making it confusingly similar to a normal 3-day viral fever.

\subsection{Anosmia and loss of smell.}

This also one of the early symptoms which in combination with flu like symptoms are high indicators of Covid-19.[12]

\subsection{Happy hypoxia}

This is one of the most unique and interesting feature so far witnessed in any disorder or disease.

Patients tends to communicate and do normal taste like they always did. Only problem occurs when their oxygen saturation levels are measure by pulse-oximeter, it shows oxygen saturation $70 \%, 60 \%, 50 \%$ or even less.[13] In such low oxygen saturation level, the person should faint or have shortness of breath. Not laughing and interacting like a person free from disease.

This is unique to SARS-CoV-2 recently disorder in mid-April 2020.

The normal oxygen saturation of body is $95 \%$ or more. When it drops to $90 \%$ or less, as seen in pneumonias people start feeling shortness of breath, fatigue, fainting etc. This might be due to fact that in pneumonia, the ability of lungs to inflate is affected. So proper gas exchange is not possible. Hence increase in $\mathrm{CO}_{2}$ are detected and prominently seen in patient. In coronavirus, it is not only the lung is affected, but more so the perfusion. The inflammation of capillaries causes clot formation due to which tissues are not fully oxygenated. But since lungs inflate and deflate properly and almost like the normal, no signs or symptoms of hypoxia are shown by the patient and patients doesn't seems to be in distress. [13]

\section{Clinical features specific to age groups}

Following are the symptoms specific to age and their mortality rate. https://www.worldometers.info/coronavirus/coronavirus-age-sex-demographics/

\subsection{0-15 year old}

Most children are asymptomatic. But they said to be carrier of the disease. Though children with underlying disease have shown severe symptoms such as heart attacks and stroke. 


\subsubsection{Kawasaki Disease}

Also known as mucocutaneous lymph node syndrome. It is a rare inflammatory childhood disorder, in which child may suffer from the following [14]:

- High fever; $\geq 40^{\circ} \mathrm{C}$

- Cough

- strawberry tongue,

- abdominal pain,

- vomiting

- swollen lymph node

- erythematous, seemingly non-pruritic, blotchy rash all over the body

Mortality: $0.2 \%$

\subsection{5-44 year old}

- Heart attacks and strokes in severe cases and person with underlying disease such as obesity, congenital heart defects and diabetes

- Usually, flu like symptoms only

- Most of them recover with no complication thereafter.

Mortality; $1 \%$

\subsection{4-65 year old}

- Severe cases heart attack and strokes

Mortality: $12 \%$

\section{4. $\geq 65$ year old}

- Highly vulnerable.

- High risk and long term complication due to severe damage to blood vessels and lungs.

Mortality: $15 \%$

\section{Possible treatment}

So far there is no cure for the disease. Young and strong people with good immunity recover from it whereas the elderly due to low immunity and low regenerative capacity suffer and face much distress and show increased mortality. Following are few symptomatic treatments given: -

- For clot formation, blood thinners such as heparin are used.

- People are advised to check their oxygen levels at comfort and secureness of their home through oximeter.

- Drugs such as Remdesivir have shown improvement.[15]

\section{Conclusion}

The new novel coronavirus is a highly contagious disease which is fatal yet preventable. People all around the world should adopt top notch hygiene practices such as washing your hands frequently and not touching face often. The road to vaccine though seems long, but it will lead to destination. Clinical trials in development of vaccine might give us new hope or we may all have to develop immunity against it. In other words, we may just have to get adapted and adjusted to it. 


\section{Compliance with ethical standards}

\section{Acknowledgments}

I thank All Mighty for completing the article. I would express my gratitude to my parents Fazal Mustafa Ali Khan and Dr. Nusrat Aziz for their valuable guidance and support in completing the article. I would also like to thank Miss Shams Fatima Khan in graphical work and Miss Sharia Fatima Khan in providing valuable information.

\section{Disclosure of conflict of interest}

The author declares that there is no conflict of interest and no financial help was taken from the institution or any other funding department.

\section{References}

[1] Ksiazek TG, Erdman D, Goldsmith CS, Zaki SR, Peret T, et al. (2003). A novel coronavirus associated with severe acute respiratory syndrome. N. Engl. J. Med. 348, 1953-66.

[2] Zaki AM, van Boheemen S, Bestebroer TM, Osterhaus A and Fouchier RAM. (2012). Isolation of a novel coronavirus from a man with pneumonia in Saudi Arabia. N. Engl. J. Med. 367, 1814-20.

[3] Myint S, Siddell S and Tyrrell D. (1989). The use of nucleic acid hybridization to detect human coronaviruses. Archives of Virology, 104(3-4), 335-337.

[4] Tyrrell DA and Bynoe ML. (1965). Cultivation of a novel type of common-cold virus in organ cultures. Br. Med. J, 1, 1467-1470.

[5] Li F. (2016). Structure, Function, and Evolution of Coronavirus Spike Proteins. Annual Review of Virology, 3(1), 237-261.

[6] Virology: Coronaviruses. Nature. (1968).

[7] Enjuanes L, Almazan F, Sola I and Zuniga S. (2006). Biochemical aspects of coronavirus replication and virus-host interaction. Annu. Rev. Microbiol, 60, 211-30.

[8] Perlman S and Netland J. (2009). Coronaviruses post-SARS: update on replication and pathogenesis. Nat. Rev. Microbiol, 7, 439-50.

[9] Zhu Na, et al. (2020). "A Novel Coronavirus from Patients with Pneumonia in China, 2019." The New England journal of medicine, 382(8), 727-733.

[10] Liu P, Blet A, Smyth D and Li H. (2020). The Science Underlying COVID-19: Implications for the Cardiovascular System. Circulation.

[11] Ackermann M, Verleden S, Kuehnel M, Haverich A, Welte T, Laenger F et al. (2020). Pulmonary Vascular Endothelialitis, Thrombosis, and Angiogenesis in Covid-19. New England Journal of Medicine.

[12] Menni C, Valdes AM, Freidin MB et al. (2020). Real-time tracking of self-reported symptoms to predict potential COVID-19. Nat Med.

[13] Couzin-Frankel J. (2020). The mystery of the pandemic's 'happy hypoxia'. Science, 368(6490), 455-456.

[14] Jones VG, Mills M, Suarez D, et al. (2020). COVID-19 and Kawasaki disease: novel virus and novel case. Hosp Pediatr.

[15] John HB, Kay MT, Lori ED, et al. (2020). Remdesivir for the Treatment of Covid-19 - Preliminary Report NEJM.

\section{How to cite this article}

Khan SF. (2020). An analysis of nCovid-19. World Journal of Advanced Research and Reviews, 6(3), 97-101. 